\title{
Geometric construction of auxetic metamaterials
}

GP. Bonneau, S. Hahmann and J. Marku

Univ. Grenoble Alpes, CNRS, Inria, Grenoble INP, LJK, 38000 Grenoble, France
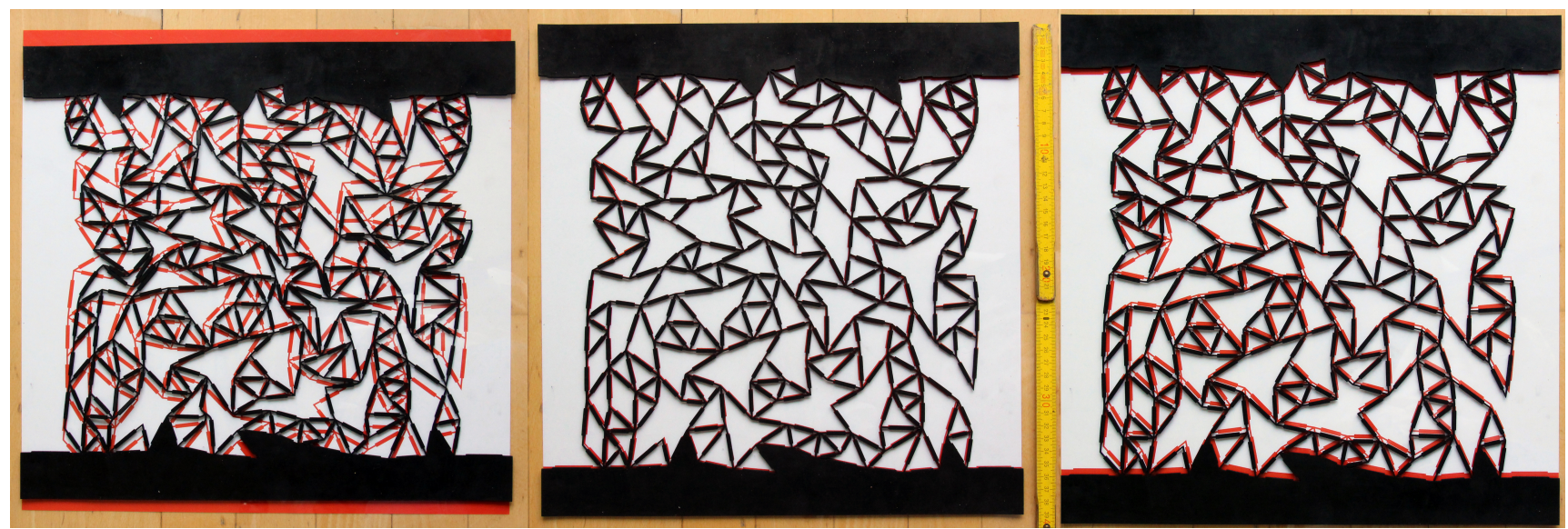

Figure 1: Vertical compression (left) and extension (right) of a laser-cut auxetic irregular network in rest state (middle) computed by our purely geometric process. Notice the horizontal, transversal contraction (left) and extension (right) characteristic of an auxetic behavior. The compressed network exhibits a Poisson's ratio $\mathrm{v}=-0.94$ for an applied strain $\varepsilon_{y}=-7.1 \%$. The extended network exhibits a Poisson's ratio $v=-0.46$ for an applied strain $\varepsilon_{y}=+3.3 \%$. Strains are applied symmetrically to the top and bottom boundaries.

\begin{abstract}
This paper is devoted to a category of metamaterials called auxetics, identified by their negative Poisson's ratio. Our work consists in exploring geometrical strategies to generate irregular auxetic structures. More precisely we seek to reduce the Poisson's ratio v, by pruning an irregular network based solely on geometric criteria. We introduce a strategy combining a pure geometric pruning algorithm followed by a physics-based testing phase to determine the resulting Poisson's ratio of our structures. We propose an algorithm that generates sets of irregular auxetic networks. Our contributions include geometrical characterization of auxetic networks, development of a pruning strategy, generation of auxetic networks with low Poisson's ratio, as well as validation of our approach. We provide statistical validation of our approach on large sets of irregular networks, and we additionally laser-cut auxetic networks in sheets of rubber. The findings reported here show that it is possible to reduce the Poisson's ratio by geometric pruning, and that we can generate irregular auxetic networks at lower processing times than a physics-based approach.
\end{abstract}

\section{Introduction}

The more you pull on a rubber band, the more it becomes thinner. Auxetic materials, however, behave counter-intuitive: they expand perpendicular to an applied stretch. The Greek word "auxetikos" meaning "what tends to increase" gives these material their name. Recent advances in digital manufacturing, where computational design, materials science and engineering meet, offer whole new perspectives for tailoring mechanical properties and fabrication of material with applications as diverse as product design, architecture, engineering and art. Auxetic materials, in comparison to standard materials, are characterized by enhanced mechanical properties such as energy absorption, indentation resistance and acoustic absorption. Promising applications range from biomedical purposes (prosthesis, blood vessels dilators, limbs, bandages), impact protection devices for sports (gloves, helmets, pads, mats), to textile for defense. They also have interesting geometric properties, 
which enable for example an initially planar object to deform out of plane into a surface with synclastic curvature. Applications here range from product design $\left[\mathrm{KCD}^{*} 16, \mathrm{Cec} 21\right]$ to architecture and art [NM15], see Fig. 2.

Auxeticity of existing synthetically created materials and structures can be demonstrated at different scales: at molecular scale $\left[\mathrm{MCM}^{*} 17\right]$, at micro-scale (polymer foams and fibers) and at macro-scale auxetic structures with a plethora of geometric structures fabricated of rubber, metal or 3D-printed (re-entrant cells, rotating units and perforated sheets).

Our focus is on the latter class of auxetic structures, also called a mechanical metamaterial. A metamaterial gains it extraordinary effective properties from rationally designed geometric structures rather than their composition. It enables to obtain new physical properties of standard materials by change of geometry. For example, a rigid wooden board can be made very flexible by cutting notches [ZEK $\left.{ }^{*} 17\right]$. And a 3D printed object made of computationally optimized microstructures can exhibit a full range of customized mechanical properties using the same printing material [SMGT18].

The auxetic structures mentioned above thus make it possible to design and manufacture auxetic metamaterials using non-auxetic materials such as metal, wood or rubber. The layout of the structure (including micro-structures) has thus a significant impact on the auxeticity. These well studied auxetic layouts, which are generally planar, some of them extended to 3D, are however all of regular geometry, composed periodically of cells and structures with axis or rotational symmetries.

In this paper the focus is on irregular auxetic structures, which have not been studied so far in geometric and computational design. In architecture new trends focus on architectural materials composed of irregular geometries, patterns or structures, e.g., the BUGA fibre pavilions made from coreless-wound fibre-reinforced polymers by Menges \& Knippers [SFS*19], the weather responsive self-forming building parts [WVMR18] or the irregular cellular structures of Schlüter and Bonwetsch [SB08]. Irregular auxetic networks may therefore enrich the variety of auxetic materials architects are interested in since a few years [MSMB17]

The fact that auxetics can be made physically of irregular structures is known since the first work on synthetically created auxetic foams by Lakes in 1987, see figures in [Lak87]. Soft matters (rubber, foams and other elastomers) made of cross-linked polymer chains are micro nodes and fibril structures and can be thought of as irregular networks represented as a graph of connected nodes. Their fabrication is very complex, including chemical and physical processes. However, the fabricated auxetic foams for example are irregular and their (re-entrant) cells clearly exhibit specific structural geometric features, which can be found analogically in many regular geometric auxetic structures.

Our idea is to exploit these features for the construction of irregular auxetic structures. That irregular auxetic structures can also be generated computationally, has been demonstrated recently in physics. Goodrich et al. [GLN15] and Reid et al. [RPW*18] compute irregular 2D networks with predefined mechanical properties, including auxetics, based of physical simulation. Inspired by their work, we propose to investigate a geometric rather than a physics-based approach by centering our method uniquely on the geometric features observed in existing regular auxetic structures. We raise the question:

Is it possible to achieve the mechanical property of auxeticity based solely on a geometric approach?

Our approach at answering this question starts with identifying the geometric properties comprising irregular auxetic structures, then developing an algorithm able to reproduce these properties on arbitrary irregular networks we generate, and finally comparing and validating our resulting networks' characteristics with known features of auxetic metamaterials. At the end we show some physical realizations of our networks clearly demonstrating auxetic behavior.

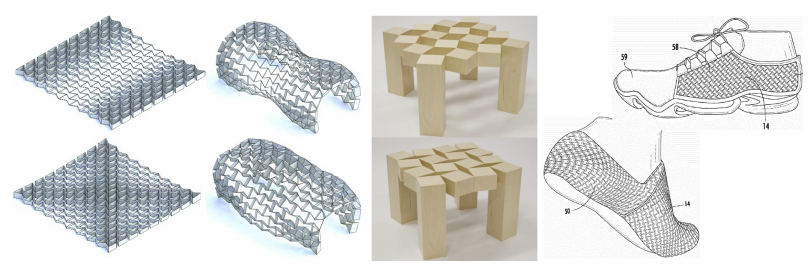

Figure 2: Bending active auxetic structures in architecture [NM15], auxetic tables [Cec21] and sport shoes with auxetic garments [Tor13] (left and middle: image courtesy of Lorenzo Mirante and Tom Cecil).

\section{Related Works}

Material science. Auxetic materials in nature are not common (crystalline materials, human tendons, skin of snakes). In 1987 Lakes [Lak87] pioneered research on auxetic materials and structures by producing the first realistic auxetic foam structure. The number of publications on auxetic materials since then has increased exponentially. One can globally distinguish between auxetic materials and geometric structures: (1) intrinsically auxetic materials, based on their chemical formulation. Here, research is still on a theoretical level, no experimental observations so far [PMW* 19]. (2) Auxetic foams are obtained by a thermomechanical process to convert conventional polyurethane (PU) foam into auxetic PU foam [Lak87, VV19]. They are expected to exhibit improved energy absorption for lighter and/or thinner components. (3) Auxetic structures for metamaterials. There are a plethora of different geometric patterns such as re-entrant structures, rotating rigid structures, chiral structures, perforated sheets, which give an auxetic effect on application of load. Review papers focus on different aspects such as the modeling, the mechanics, or the deformation mechanisms [RDT*18, KZ17, Lim17, GGLR11]. Origami structures also qualify as auxetic mechanical metamaterials. Their mechanical behavior is largely driven by the crease patterns, folding sequences, and other parameters describing the geometry [YY15, KME* 17]. The Poisson's ratio for the aforementioned purely geometric and origami structures can be derived explicitly. Elipe et al. [EL12] present a comparative study of most known 2D and 3D auxetic geometries using CAD modeling, tetrahedral meshing and FEM simulation. (4) Auxetic composites and textiles [ZJH16]. 
Our approach takes inspiration from both, structural geometric features (3) and the strategy employed for foam fabrication (2): we propose an algorithm which modifies conventional networks by iteratively removing node connections (edges in a graph) following some geometric criteria such that they exhibit auxetic behavior under certain loading conditions.

Computational design of mechanical metamaterials. The computational design and digital fabrication of physical objects attracts a growing interest in computer graphics. While the aforementioned geometric auxetic structures were originally conceived geometrically, computational approaches relying on, e.g., topology optimization, enable to automatically generate them to yield new ones. Our focus here is only on computational design and optimization methods including auxetic metamaterials. Zhou et al. [ZSCM17] focus on the generation and characterization of databases of microstructures with maximal material property coverage and thus discover multiple new 3D auxetic microstructures. Schumacher et al. [SMGT18] show that computationally optimized microstructures can exhibit a full range of customized mechanical properties (bending stiffness, anisotropy, auxeticity). The design of tileable and printable microstructures taking into account manufacturing constraints have been investigated by Panetta et al. [PZM*15]. Martinez et al. [MSS*19] generate 2D tile geometries with a graduation of mechanical properties computed as Voronoï diagrams of regular lattices under star-shaped distance functions. Topology optimization allows to generate new design of auxetic metamaterials with prescribed non-linear properties computationally [WSJ14, ALS14, VCW*17, ZK19, ACN20]. These various approaches underline the growing interest in auxetic structures in the computer graphics community. The goal of the present work is to initiate the exploration of irregular auxetic networks from a geometric point of view.

Geometric design of mechanical metamaterials. [JTV* 15$]$ present a geometric approach for design and optimization of polyhedral patterns (possibly auxetic) onto 3D surfaces based on affine symmetries. [KCD*16, KLPCP18] propose a design tool based on conformal mappings to generate and fabricate more complex shapes with a purely geometric approach using a rotating auxetic structure. FlexMaps [MPI*18] is an inverse modeling approach with an auxetic chiral structure to design and fabricate smooth $3 \mathrm{D}$ surfaces. All these methods exploit repetitive regular patterns to be mapped onto a doubly-curved surface.

Irregular microstructures with prescribed mechanical properties are generated by [MDL16] and [TTZ*20]. [MDL16] produces stochastic, aperiodic graded open-cell Voronoï foams with prescribed Young's modulus. The resulting material is however limited to an almost stable positive Poisson's ratio of about +0.3 . We conjecture, that the convexity of Voronoï cells prevents auxetic behaviors. [TTZ*20] also relies on convex structures to produce materials which are rigid in one direction and compliant in transverse directions. While our material is of similar stochastic nature, we seek to generate auxetic material by favoring the occurrence of concave structures.

Auxetics are known to be attractive also in architecture not only for their mechanical properties, but for aesthetics as well [MSMB17]. Architecture is demanding for irregular structures, as the examples given in the introduction show. Whereas conventional foam serves in the M.ANY project of Schlüter and Bonwetsch [SB07] as an ideal model for architectural form finding, our approach draws inspiration from auxetic foams and seeks to reproduce their irregular pattern.

Irregular auxetic structures. While auxetic geometric structures consisting of ordered or periodic networks are well studied, the effect of disorder is much harder to conceive and to study. A first approach is to start from the regular honeycomb structure and to perturb it by randomly displacing the vertices [HSS*09]. The resulting network may not be auxetic anymore. A heuristic search strategy is employed, which computes at each iteration 70 perturbed networks and applies a FEM simulation to each (including CAD model + tetmeshing). The one with lowest Poisson's ratio is selected for the next iteration. 800 iterations with thousands of FEM simulations are performed, which is excessively time consuming. Without any guarantee of convergence, experiments show that the resulting structures are auxetic in mean with values ranging within $[-0.65,+0.2]$. Other approaches perturb the vertex positions of a less regular Voronoi cell structure [HPAZ18]. The heuristic process of finding the optimal vertex positions and a mass-spring simulation with linear and angular springs allows to select among a huge set of networks the most auxetics ones. A theoretical formulation of irregular auxetic honeycombs with spatially random variations in cell is proposed in [LWHZ14]. All these structures are mappings of the regular conventional or re-entrant honeycombs. They present geometric irregularities, but topologically they are identical to the honeycombs with all vertices of order 3 .

In $\left[\mathrm{RPW}^{*} 18, \mathrm{RPB}^{*} 19\right]$, the authors show that irregular auxetic networks of arbitrary topology can be obtained by initializing a network from a jammed sphere packing simulation, and iteratively pruning edges selected from their contributions to the elastic moduli (bulk, pure and shear moduli). This approach requires computationally intensive physical simulations at each step of the process. While we follow a similar path in which we start with an initial network and iteratively prune edges in it, we rely exclusively on simple geometric criteria for doing so. A single physical simulation is required at the end of the process for computing the actual Poisson's ratio of the network.

\section{Technical Contributions}

\subsection{Summary of contributions}

The goal of our work is to compute irregular auxetic structures through pruning of initial irregular networks. But instead of relying on multiple physical simulations at each pruning step, our idea is to seek a final network exhibiting specific geometric features. We therefore stick to a geometrical approach throughout our process. A physical simulation is only required at the end in order to determine the effective actual Poisson's ratio of the resulting network. The challenge we face is to develop a purely geometrically guided pruning process. We will show that our pruning algorithm can produce networks with reduced Poisson's ratio and that we achieve negative Poisson's ratios for almost all our networks. We will further show, that the proposed algorithm succeeds in less than a few seconds for the whole pruning process, compared to minutes to hours for previous algorithms relying solely on physical simulations. 
Our contributions can be summarized as follows:

1. analysis of geometric features in auxetic networks (Sect. 3.4)

2. introduction of two geometric measures for detecting these features in irregular networks (Sect. 3.7)

3. a pruning algorithm guided by these new geometric measures (Sect. 3.7)

4. a vertex displacement procedure increasing auxeticity (Sect. 3.8)

5. computation of the Poisson's ratio for the resulting irregular networks (Sect. 3.9).

In addition to these technical contributions, we also present in section 4.2 an extensive validation of our results and show some physical realizations.

\subsection{Notations}

An irregular network is modeled as a unoriented graph $G=(V, E)$ connecting planar vertices $v \in V \subset \mathbb{R}^{2}$ by edges $e=\left\{v_{1}, v_{2}\right\} \in$ $E, v_{1}, v_{2} \in V$. To conform with our focus on geometric processing, we favor the terms vertices and edges instead of particles and bonds which are common in the material and physics literature.

\subsection{Poisson's ratio}

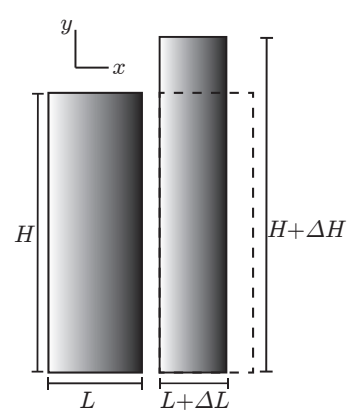

When a sample of material under tension or compression along one direction, it deforms in the orthogonal direction. This material behavior is charaterized by the Poisson's ratio denoted $v$ and named after Siméon Denis Poisson (1787-1840)[Poi33]. If we consider a piece of material and align it to the $x$ and $y$ axis, see the inset, the Poisson's ratio between transverse strain $\varepsilon_{x}$ and longitudinal strain $\varepsilon_{y}$ in the elastic loading direction $y$ is defined by

$$
v=-\frac{\varepsilon_{x}}{\varepsilon_{y}}=-\frac{\Delta L / L}{\Delta H / H}
$$

For isotropic 2D materials, $v$ does not depend on the loading direction and ranges between $-1<v<0.5$ in 3D, between $-1<v<1$ in $2 \mathrm{D}$. Values far below -1 can be achieved if the material is allowed to deviate from isotropy. Usual materials have positive Poisson's ratio, i.e. when stretched in one direction they shrink in orthogonal directions (e.g., incompressible rubber 0.5, metal 0.3, foam 0.1-0.5 and cork 0). Auxetic materials are defined by a negative Poisson's ratio. If stretched longitudinally, they stretch also in the transverse direction, as illustrated in Fig. 3.

\subsection{Geometric features of $2 D$ auxetic networks}

In this section, we analyze salient geometric features shared by all standard periodic 2D auxetic networks, reviewed for example in [RDT*18]. Interestingly the same geometric features can be observed in irregular auxetic networks [RPB*19].
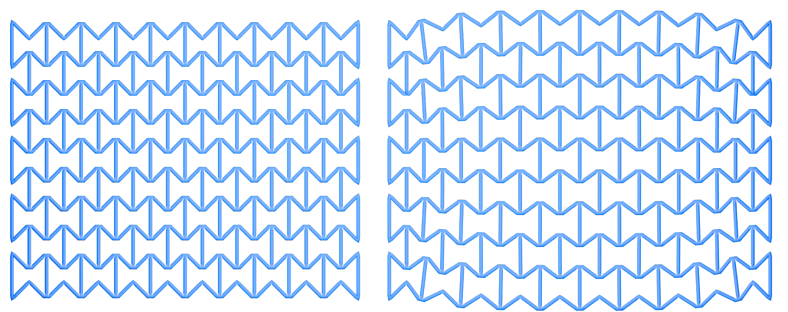

Figure 3: Horizontal traction of a re-entrant honeycomb network. Reference configuration (left), loaded configuration with uni-axial strain $\varepsilon_{x}$ of $10 \%$ (right) and Poisson's ratio $\mathrm{v}=-0.8$.
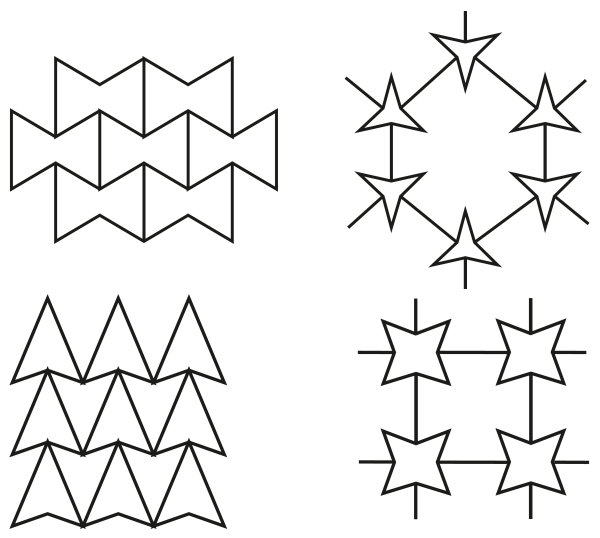

Figure 4: re-entrant periodic auxetic networks.

\section{Coordination number}

The coordination number, denoted $Z$, is a physical quantity measuring the number of bonds connecting particles in a system. For example in the context of jamming of spheres [LN10], it is the average number of spheres in contact with one given sphere. In our geometrical setting, where the networks are modeled as planar graphs (see Section 3.2), the coordination number is the average degree of vertices in the graph. In dimension $d$, the minimum coordination number to maintain rigidity of a disordered system, called isostatic value, is $Z_{\text {iso }}=2 d$ [GLN15]. A value of 6 , which is the average degree of vertices in a planar triangulation, is well above the isostatic value, and can not lead to auxetic networks [LNTX19]. According to $\left[\mathrm{RPW}^{*} 18\right]$ networks with lower coordination numbers are more amenable to physics-based pruning. Networks pruned from $Z_{0}=5.2$ lead to the lowest value of $v$ in their study. Regular periodic networks such as honeycomb, n-STAR and arrowhead shown in Fig. 4 have even coordination number below the isostatic value of 4 in $2 \mathrm{D}$.

In our approach, the coordination number $Z$ decreases at each edge pruning and is thus well suited to control the process. We will use $Z$ as a stop criteria for the pruning algorithm. Note that for $Z<3.0$ the network is so sparse that the physical simulation for applying the deformation and measuring $v$ becomes unstable. In the results Section 4 we study the dependency of the Poisson's Ratio from the coordination number (see Fig. 14). 


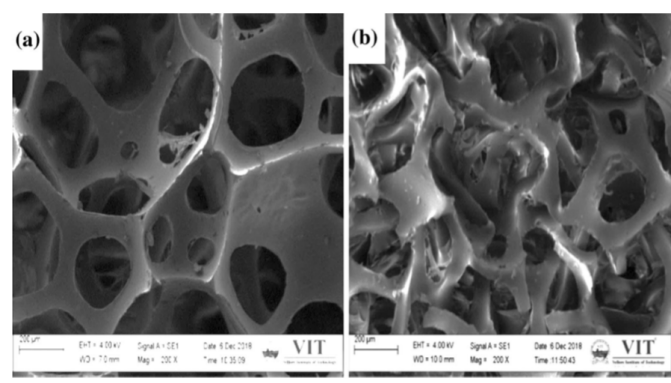

Figure 5: micro-structure of synthetic foams: (a) conventional polyurethane foam, (b) auxetic polyurethane foams in contrast to the conventional foam [VV19]. The auxetic foam exhibits concave cells. Image courtesy of Springer Nature.

\section{Pointed vertices}

A re-entrant vertex, also referred to as pointed, is a vertex which has all its neighbor vertices in the same halfplane. In the regular examples illustrated in Fig. 4, all vertices are pointed. There is one angle larger than $\pi$ between consecutive edges around a pointed vertex. In our pruning algorithm developed in Section 3.7 we define the index of pointedness and angle of pointedness for arbitrary vertices, and use these measures in order to guide to pruning process.

\section{Concave polygons}

The pointed vertices manifest as concave angles in polygons within the network, as noticeable in Fig.4. These polygons tend to collapse inward at pointed vertices when compressed. A sufficient number of such polygons can lead to globally auxetic behavior. Concave cells can also be observed in auxetic synthetic foams, as illustrated in Fig. 5. In [RPB*19], the authors observe that the network geometry changes during pruning to create concave polygons. They show that the percentage of concave polygons correlate quite well with $v$. Our pruning algorithm also creates pointed vertices (see Sec. 3.7). Furthermore, the vertex displacement procedure introduced in Section 3.8 exaggerates the concavities of these polygons.

\subsection{Overview}

Our method for creating auxetic irregular networks is built upon the geometric features discussed previously. It is composed of 4 steps:

(1) Generation of an initial dense random network, using Poisson Disk Sampling and Delaunay triangulation.

(2) Pruning of this network, based on local geometry criteria. We introduce two new measures which guide the pruning with the goal of obtaining final networks exhibiting the geometric features.

(3) Modification of the vertices position, which allows to significantly improve the auxetic behavior of the pruned network. Here again, the local geometry governs the process.

(4) Computation of the Poisson's ratio, by applying a physicsbased simulation. We use a non-linear mass-spring system for computing a deformation of the network resulting from uni-axial loading.

A set of pruned networks exhibiting the geometric features of auxetic networks can be generated with our method in less than a second for each network. Only local geometrical criteria are used throughout steps (1) to (3). The final computation of the Poisson's ratio in step (4) allows to measure the actual auxeticity of the network. Fig. 6 illustrates steps (1) to (3).
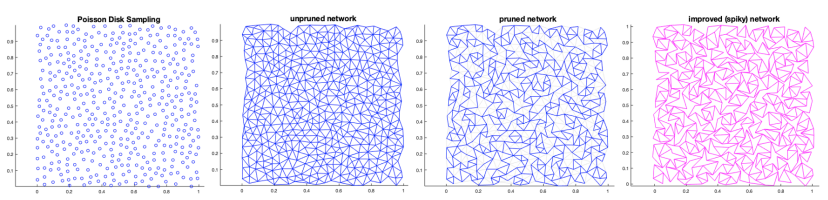

Figure 6: Overview: (a) randomly generated vertices using Poisson Disk Sampling (Section3.6). (b) Initial network generated using Delaunay triangulation and removal of boundary edges (Section3.6). (c) pruned network (Section3.7). (d) improved network obtained by vertex displacements (Section3.8).

\subsection{Initial networks}

In $\left[\mathrm{RPW}^{*} 18\right]$ the initial networks are produced by simulating a jammed packing of frictionless spheres at zero temperature, following the work of [LN10]. Instead of using a computationally intensive simulation we choose to apply a combination of Poisson Disk Sampling and Delaunay triangulation, which produce similar well balanced networks. Blue noise sample patterns - for example produced by Poisson Disk Sampling, where all samples are at least distance $r$ apart for some user-supplied density parameter $r$ - are generally considered ideal for many applications in rendering [Bri07]. We use this type of sampling to generate the vertices of our initial network. The algorithm presented by Bridson is extremely efficient as it takes $O(N)$ time to generate $N$ samples. The result of this algorithm is a set of randomly allocated vertices positioned at a minimal $r$ distance from each other. In order to finalize the network, we apply a Delaunay triangulation to connect the generated Poisson Disk samples and remove long boundary edges. This can also be efficiently computed in $O(N \log (N))$. Fig. 6 shows the result of Poisson Disk Sampling (a) and Delaunay triangulation (b).

\subsection{Pruning Algorithm}

Initial networks have well distributed vertices within a given domain. We now explain how to transform this initial network in a new network that exhibits the geometric features of auxetic networks established in Section 3.4. We do so by iteratively pruning (removing) edges from the initial network, in such a way that the final network exhibits the geometric features. In particular we seek to obtain networks with a high percentage of re-entrant vertices. To this end, we introduce two new measures, which we name index of pointedness (IoP) and angle of pointedness.

Definition. The index of pointedness $\mathbf{n}$, of a vertex $\mathrm{v}$, is the minimum number of consecutive edges $e_{i}$ for $i=1 \ldots n$ incident to $\mathrm{v}$, necessary to remove in order to attain a pointed vertex. A vertex having an index of pointedness $n$ is referred to as n-pointed. For each such set of consecutive edges, the angle of pointedness is the single angle larger than $\pi$ that would appear by removing them. 
These two measures are illustrated in Fig. 7. Based on this definition, a vertex is pointed iff it has an index of pointedness $n=0$. Notice that the angle of pointedness depends on the sequence of consecutive edges whose removal would make the vertex pointed. Pruning any single edge around a vertex with an index of pointedness larger or equal to 2 will not make it pointed, as illustrated in Fig. 7(c). The index of pointedness enables to characterize the mesh vertices being the most amenable to increase the percentage of pointed vertices when one of their incident edges is removed. Our method revolves around this concept as follows.
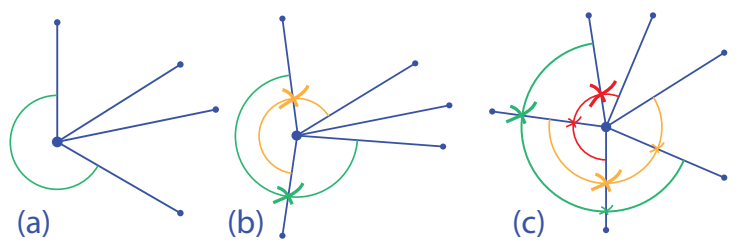

Figure 7: Index and Angle of Pointedness. 0-pointed (a), 1-pointed (b) and 2-pointed (c) vertices. The edges to be removed in order for the vertex to become 0-pointed are color coded (same colored $x$ on consecutive edges to remove). The circular arcs visualize the angles of pointedness, smallest angle in red, mid angle in orange and largest angle in green.

Algorithm 1 reduces the coordination number $Z$ by iteratively pruning edges, until a target coordination number is reached. Edges are selected for pruning such that the indices of pointedness of vertices gradually decrease. Among all such edges we favor those with the largest angle of pointedness.

In order to maintain a connected structure and topological coherent network we do not remove an edge if any of its incident vertices has a degree below a given value. We have noticed experimentally that a minimum degree of 2 works for our purpose. A vertex of degree 1 in the network is clearly not desirable since it would result in edges or polylines sticking out of polygons. Furthermore by allowing vertices of degree 2 , "hanging" polygons could in theory appear, that would be connected to the rest of the network via a single vertex of degree 3 . Such structures are also not desirable since they wouldn't contribute to the auxeticity of the network. Though theoretically possible, we didn't encounter this situation in our experiments. Indeed the random selection of edges in our algorithm, and the fact that it gradually decreases the indices of pointedness result in a pruning process that is well distributed within the domain, and that reaches the target coordination number $Z$ before hanging polygons appear.

\subsection{Vertex displacement}

The pruning algorithm introduced in Section 3.7 produces networks exhibiting a high percentage of pointed vertices and concave polygons. In the present Section we introduce a procedure that increases the angle of pointedness (see Sec. 3.7) of the resulting networks, by modifying the position of vertices. Though simple, this procedure proves nevertheless to be very efficient for decreasing the Poisson's ratio, as validated in Section 4.2 .

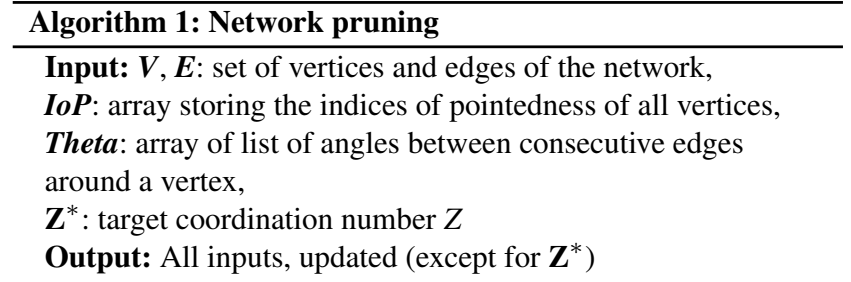

Compute coordination number $Z$

while $\underline{Z>Z^{*}}$ do
pick a randomly chosen vertex $v$ in the set $V$

\section{if $\operatorname{IoP}(v)>0$ then}

Select all sequences of consecutive edges that can make $v$ a pointed vertex (see Fig. 7)

Using Theta, select the sequence of consecutive edges maximizing the angle of pointedness

Let $e$ be the first edge this sequence (in cw order)

Let $w$ be the vertex incident to $e$ distinct from $v$

if $\frac{\text { degree }(w)>2}{\text { Remove edge } e}$ then
end if

Update $V, E, I o P$, Thet $a$ and $Z$

end if

end while

return $V, E, I o P$, Theta

In order to displace the vertices, we compute a displacement vector, which is the normalized sum vector of the vectors formed by the edges incident to the vertex of interest. We use a scalar coefficient $\lambda$ as a parameter for regulating the amount of displacement. Referring to Figure 8, the new vertex position $v^{\prime}$ is computed by:

$$
v^{\prime}=v-\lambda \frac{\sum_{i} v_{i}-v}{\left\|\sum_{i} v_{i}-v\right\|}
$$

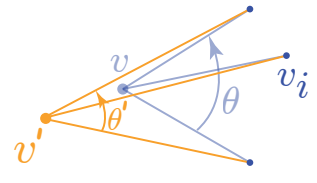

Figure 8: A pointed vertex before (blue) and after (orange) displacement. vertices. In that case, all the vectors connected to the vertex of interest lie on one half-plane and the vertex is translated in the other half-plane. This vertex displacement is therefore particularly effi- 
cient when applied after pruning since pruning increases the ratio of pointed vertices. We apply this procedure to all vertices independent whether they are pointed or not. Fig. 12 shows a pruned network (b) and the spiky network (c) resulting from the application of vertex displacement. In Fig. 17, we show that the vertex displacement has little to no effect if applied to a network before pruning.

\subsection{Poisson's ratio computation}

Let us first recall that one of our main concerns is to favor as much as possible purely geometric processing and to avoid physical simulations which are computationally demanding. Nevertheless, we still need a physical simulation in order to compute the Poisson's ratio of the networks we produced, for evaluation purposes.

In order to simulate the physical response of the networks we generate, we model them via a mass-spring system. We considered networks with linear and angular springs, as detailed below. Let us denote $v^{\text {rest }}$ the position of a vertex in an unloaded network, and $v$ its new position in the deformed network under physical loading. A linear spring for an edge $\left\{v_{1}, v_{2}\right\}$ holds a potential energy $E_{L}$ :

$$
E_{L}\left(v_{1}, v_{2}\right)=\frac{1}{2} K_{L}\left(\frac{d-d^{\text {rest }}}{d^{\text {rest }}}\right)^{2} d^{\text {rest }},
$$

where $d=\left\|v_{2}-v_{1}\right\|$ and $K_{L}$ the stiffness coefficient for the linear springs. The potential energy of an angular spring $E_{A}\left(v, v_{1}, v_{2}\right)$ is given by

$$
E_{A}\left(v, v_{1}, v_{2}\right)=\frac{1}{2} K_{A}\left(\theta-\theta^{\text {rest }}\right)^{2}\left(\frac{d_{1}^{\text {rest }}+d_{2}^{\text {rest }}}{2}\right),
$$

where $d_{i}=\left\|v_{i}-v\right\|, i=1,2, \theta$ is the angle between neighboring edges $\left\{v, v_{1}\right\}$ and $\left\{v, v_{2}\right\}$, and $K_{A}$ is the stiffness coefficient for the angular springs. For each vertex $v$ with adjacent vertices $v_{i}, i=0 \cdots n-1$ we insert the angular springs $\left(v, v_{i}, v_{i+1} \bmod n\right), i=$ $0 \cdots n-1$. Note, that $K_{A}$ has to be chosen some orders of magnitude lower than $K_{L}$ in order to favour the hinged vertices to rotate. In our simulations, we set $K_{L}=20$ and $K_{A}=0.001$. The physical simulation consists in computing the position of the deformed vertices $v$ that minimize the total potential energy $E_{\text {tot }}$ under stress. $E_{\mathrm{t} o t}$ is defined as the sum of a compressive component (3) and a bending component (4). The IpOpt optimization library [WB06] is used to solve the non-linear problem. Fig. 13 shows results of three uni-axial vertical traction simulations, as well as the distribution of the optimal potential energy $E_{\text {tot }}$ across all edges.

As boundary conditions (BC), we enforce uni-axial strains in either $x$ or $y$ direction in our simulations. They are implemented by translating along the loading direction the vertices within a small ribbon at the top and bottom of the network. We use a width of $10 \%$ of the height for these ribbons. This type of strong BC, which prevents the network to deform in transverse direction inside these ribbons, is easy to implement in physical experiments. On the other hand, in order to evaluate the Poisson's ratio accurately, one has to measure the transverse deformations far from the $\mathrm{BC}$. We do so by observing the induced displacements of three points at the four extremities within central vertical and horizontal ribbons. The width of these central ribbons is $20 \%$ of the network height. As an example, look at Fig. 9: uni-axial vertical strain $\varepsilon_{y}$ of $3 \%$ is enforced in $y$-direction. Let $x_{l}$ and $x_{r}$ be the average horizontal coordinates of the three leftmost and three rightmost vertices, then the horizontal strain is measured as $\varepsilon_{x}=\frac{\left(x_{r}-x_{l}\right)-\left(x_{r}^{\text {rest }}-x_{l}^{\text {rest }}\right)}{\left(x_{r}^{\text {rest }}-x_{l}^{\text {rest }}\right)}$. $\varepsilon_{x}$ is negative for a horizontal contraction and positive for a horizontal expansion. Using the formula (1), we get the Poisson's ratio for uni-axial vertical loading with $v=-\frac{\varepsilon_{x}}{\varepsilon_{y}}$ and for horizontal loading with $v=-\frac{\varepsilon_{y}}{\varepsilon_{x}}$.

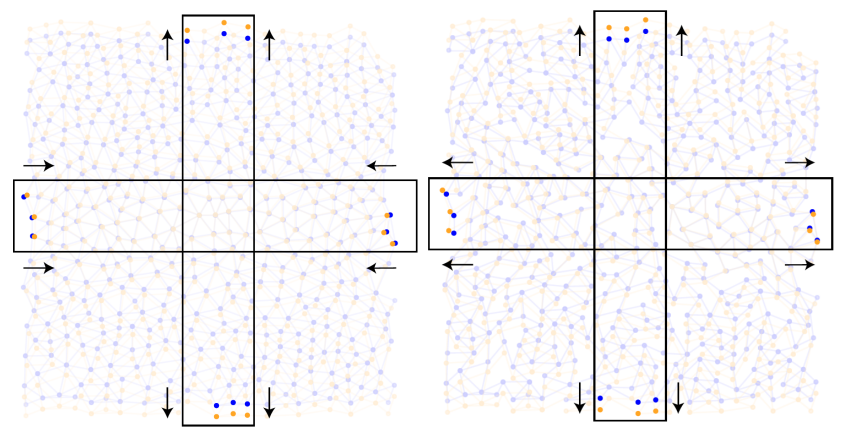

Figure 9: Enforcing a vertical expansion in two networks. A resulting horizontal contraction indicates a positive Poisson's ratio (left). A horizontal expansion indicates an auxetic network (right). Blue (resp. orange) vertices correspond to the unloaded (resp. loaded) network. The highlighted vertices are used to measure the horizontal and vertical strains.

Note that it is possible to measure the Poisson's ratio on the full structure, if the constrained vertices are allowed to slide along the direction transverse to the load. This means, that for the $y$-strain, only the y displacement component of the top/bottom nodes are constrained. While these relaxed boundary conditions are easy to implement in simulations, they are difficult to reproduce in physical experiments. Our stronger BC on the other hand, allows to compare experimental realization (described at the end of section 4.2) with simulation, as illustrated in Fig. 10.
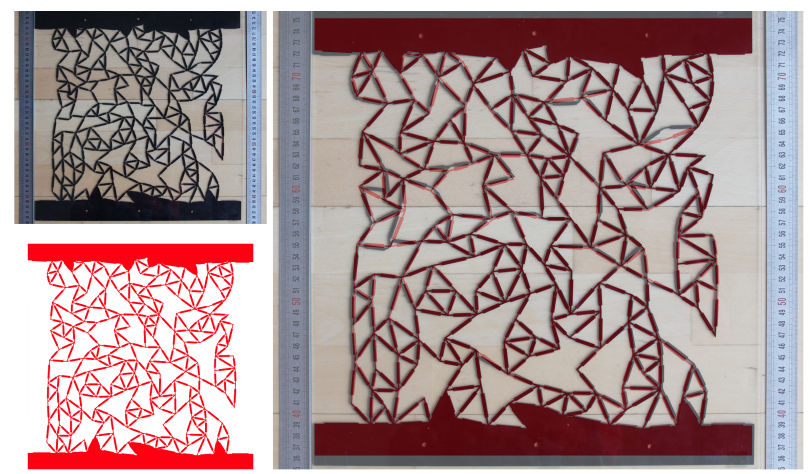

Figure 10: Matching of simulation and physical realization. Network (same as in teaser) extended in the vertical direction with a strain of 6\%. Physical realization (top left), simulation (bottom left), superimposed physical realization and simulation (right) 


\section{Results}

\subsection{Methods \& Experiments}

The computation of the irregular auxetic networks has been implemented in MATLAB, computation of the Poisson's ratio in $\mathrm{C}++$ based on IpOpt library. Experiments were run on a light MacBook Air (2018) with 1.6GHz Intel Core i5 processor running at $1.6 \mathrm{GHz}$ with $16 \mathrm{~GB}$ RAM.

In order to enable comparison between results obtained with different parameters it is therefore necessary to use for each experiment a large set of randomly generated input networks. We use sets of 100 networks with $\approx 460$ vertices each.

In all our results we use the following color coding. The networks resulting from pruning with Algorithm 1 (Section 3.7) are called pruned networks and shown in blue. The unpruned or initial input networks are shown in the same blue color. $Z_{0}$ and $v_{0}$ are respectively their coordination number and Poisson's ratio. The networks obtained from the vertex displacement procedure (Section 3.8) are called improved or spiky networks, due to their "spiky" look, and shown in pink.
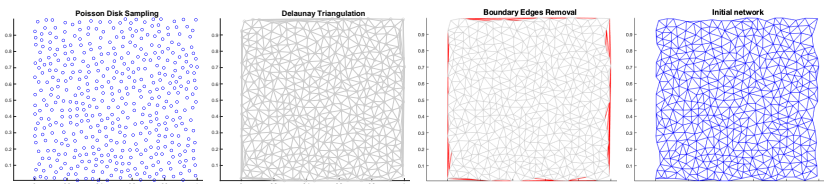

Figure 11: Initial random network in 4 steps: (a) Poisson Disk Sampling with 450 points. (b) Delaunay Triangulation. (c) Removal of long boundary edges. (d) Initial network - input for pruning algorithms.

\subsubsection{Initial networks}

We generate our initial networks using Poisson Disk Sampling (Section 3.6) in order to get evenly distributed vertices, see Fig. 11. For all networks in this chapter we used an implementation from [Sum19] with parameters $N=30$ and maxAttempt $=2.5 * \log (N)$ which gives us random initial networks with roughly 460 vertices $\mathbf{v}_{i} \in[0,1] \times[0,1]$. The fabricated networks are obtained with $N=20(\sim 200$ points $)$ and $N=15(\sim 115$ points $)$. A Delaunay triangulation is then applied to the set of points. All our initial networks have

$$
\mathbf{Z}_{\mathbf{0}}=\mathbf{5 . 6 9},
$$

which guarantees us equal starting conditions for the numerical and practical experiments.

\subsubsection{Physics-based simulation}

Subsequently, we need to test the auxetic nature of the resulting networks by simulating their elastic behavior under tensile or compressive loading conditions. The Poisson's ratio in this section is computed following the procedure presented in Sect. 3.9 using the stronger boundary conditions. We also implemented the relaxed $\mathrm{BC}$ and found almost the same PRs. They were slightly smaller;
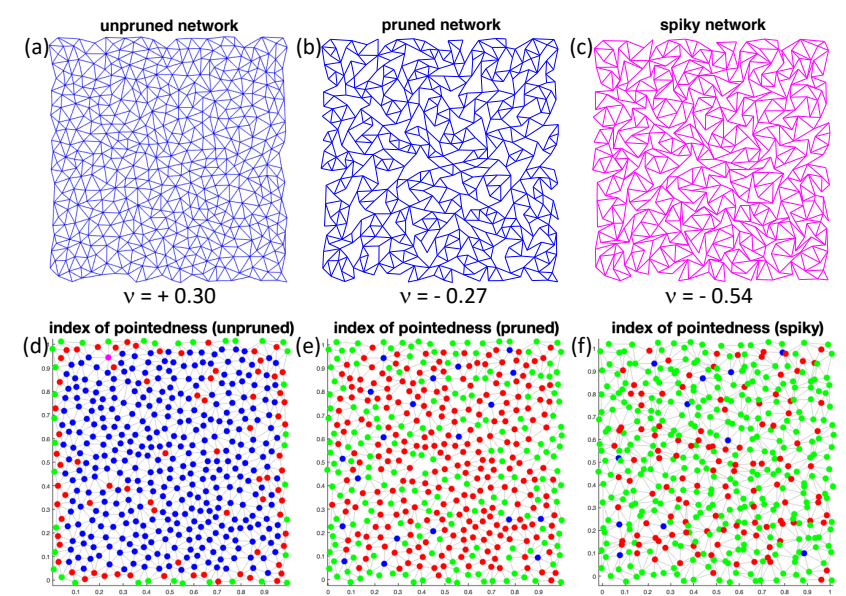

PN ratio $8 \%$

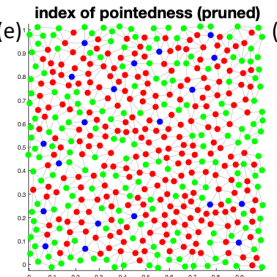

PN ratio $41 \%$

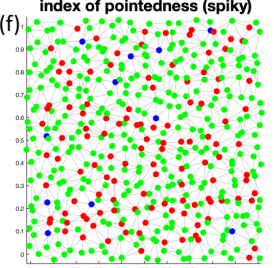

PN ratio $71 \%$

Figure 12: (a) initial network with 458 vertices, $Z_{0}=5.69 .(b)$ pruned network with $Z=4.0$ and $v=-0.27$. (c) spiky network with $\mathrm{v}=-0.54 .(d)-(f)$ show as color plot the index of pointedness (IoP) for all vertices in the network, $I o P=0$ in green, $I o P=1$ in red, $I o P=2$ in blue. The ratio of pointed vertices with $I o P=$ 0 increases from 0.08 for the initial network, 0.41 for the pruned network to 0.71 for the spiky network.

0.03 is the mean difference. To this end, an uni-axial strain in $x$ or $y$-direction with an amount of $3 \%$ is applied and the simulation minimizing the total potential energy is launched. The choice of the spring stiffness $K_{L}$ and $K_{A}$ has an influence on the mechanical behavior of our networks. Indeed, by choosing low stiffness values, the material becomes more flexible. However, attention has to be paid, since auxeticity should not be achieved because of lack of stiffness in the simulation. We calibrated these parameter to $K_{L}=20$ and $K_{A}=0.001$ so that our simulations and the computed Poisson's ratios are coherent with the literature. In [OIBB $\left.{ }^{*} 11\right]$ it is reported, that arbitrary planar triangulations exhibit a Poisson's ratio of $v=0.3$. Experimental observation by Lakes [Lak91] confirm that most common isotropic materials exhibit Poisson's ratios close to $1 / 3$. All our unpruned networks have indeed $v_{0} \approx 0.3$, see the blue horizontal line and blue dots with small standard deviation (STD) in Figures 17, 14, and 18 thus validating the choice of these stiffness parameters.

\subsection{Validation}

Results. Before explaining our results let us start by showing just one example output of our algorithm in Fig. 12. In the top row, we see for a target $Z=4.0$ a pruned network with negative Poisson's ratio $v=-0.27$ and a spiky network with negative $v=-0.54$. The bottom row visualizes with different colors the index of pointedness. Blue dots correspond to 2-pointed vertices, red dots correspond to 1-pointed vertices. And the green dots are the 0-pointed vertices, also called pointed or re-entrant. These are the vertices our algorithm is supposed to increase the percentage of. In this example, the percentage of pointed vertices in the network increases from $8 \%$ (initial network) to $41 \%$ (pruned network) up to $71 \%$ for the spiky network. 

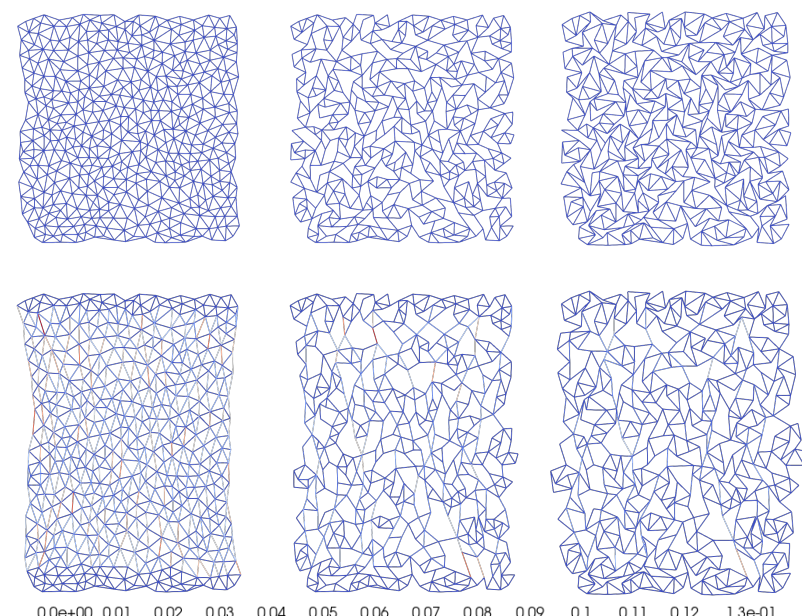

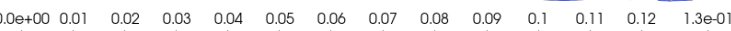

Figure 13: Uni-axial vertical traction simulation of an unpruned network with $Z=4$ (left), the corresponding pruned network (middle) and its spiky displacement (right). The top-row shows the unloaded networks. The bottom row shows the loaded networks with colors indicating the distribution of the total potential energy $E_{t o t}$ (see Sect. 3.9).

In Fig. 13 we show the deformation behavior of one selected network in order to highlight the difference between an unpruned network with positive Poisson's ratio (left column) and auxetic networks (middle and right column). The auxetic behavior of the spiky network shown in Fig. 13(right) is clearly visible. The metamaterial expands in $x$-direction under tensile loading in $y$-direction. The typical "hourglass" behavior of a standard material with positive Poisson's ratio $v=0.3$ can be observed for the unpruned network (left). The pruned network (middle) is also auxetic but to a smaller extent.

As explained above, for different values of the target coordination number $Z$, we computed a set of 100 networks. Each network was then deformed by applying an uni-axial strain in y-direction to the top and bottom border vertices of the network. The result of such an experiment for $Z=4$ is shown in Fig. 14. One observes that the Poisson's ratios for the individual networks belong to an interval following the probability distribution $P(v)$ (left). The individual $v$ values are plotted (right). We have conducted this experiment several times for 100 networks with the same $Z$, the result gives always the same probability distribution of $v$. The spiky networks are almost all auxetic with quite strong negative Poisson's ratios. The pruned networks are in mean close to $v=0$, but in all cases, the Poisson's ratio is smaller than for the initial network.

We can conclude that our geometric pruning algorithm, even though purely geometric without any intermediate feedback from a physics-simulation about evolution of the Poisson's ratio, is effective in reducing the Poisson's coefficient and that the spiky versions are remarkably auxetic. In particular our spiky networks with very few non-auxetic specimen compare favorably to previous work by [HSS*09] who achieve a similar mean Poisson's ratio with a worse
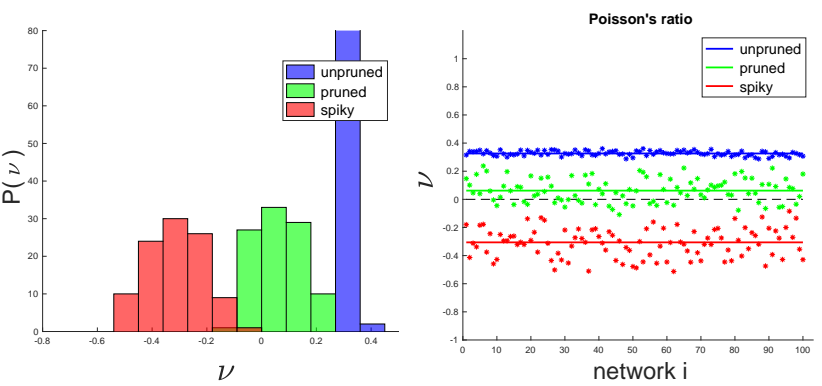

Figure 14: Probability distribution of $\mathrm{v}$ for 100 pruned networks with $Z=4.0$ and their improved (spiky) versions (left). Strains for both deformations are $\varepsilon_{y}=0.03$. Individual plots of $\mathrm{v}$ and mean values as horizontal lines (right).

STD at the cost of thousands of physics-based simulations (timing are unfortunately not provided). In the following, we will analyse the results and validate the method in more detail.

Choice of $Z$. The coordination number $Z$ in our approach is the stopping criteria of the pruning Algorithm 1. $Z$ is monotonically decreasing during pruning, since each removed edge reduces the degree of its two vertices by 1 . We found the optimal $Z$ by conducting the following experiment. For 8 equidistant values $Z \in[3.0,3.2,3.4,3.6,3.8,4.0,4.2,4.4]$, we computed a set of 100 pruned and spiky networks and evaluated the mean Poisson's ratio. For the three types of networks, unpruned, pruned, spiky, we reported the mean $v$ as a dot in Fig. 15. The optimal value for which the spiky network has minimal Poisson's ratio with greatest probability is $Z=4$.0. For the pruned networks, $Z=3.6$ is best. Pruned networks with lower $Z$ exhibit coarser and thus more flexible networks than for bigger $Z$. However, reducing $Z$ below 3.6 or 3.4 does not reduce $v$ but tends towards unstable networks. Massspring networks with angular restraints loose rigidity below $Z=3$, see Section 3.4. In conclusion, we recommend to use $Z \in[3.6,4.0]$.

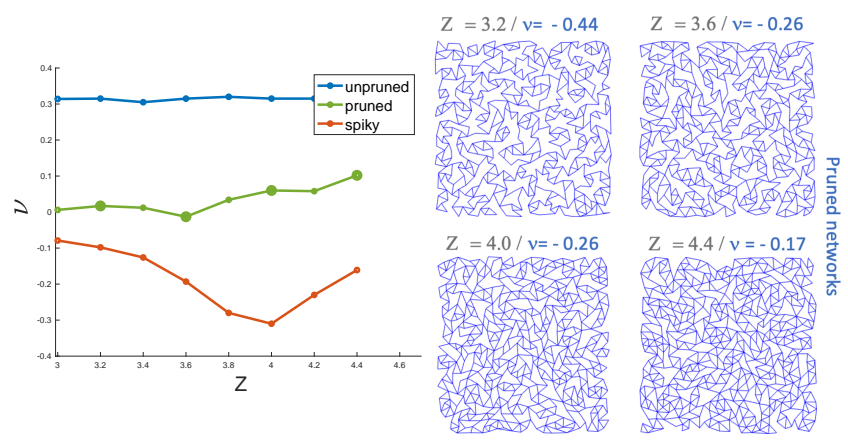

Figure 15: Poisson's ratio resulting from pruning 100 networks with different target $Z$ and $\varepsilon_{y}=0.03$. Left: mean Poisson's ratios are plotted for the initial unpruned networks (yellow), pruned networks (blue) and optimized spiky networks (orange). A minimum $\mathrm{v}$ is observed at $Z=4.0$ for the optimized networks. Right: example networks for different $Z$ values. 
Evolution of Poisson's ratio. Let us now investigate the central question about the evolution of the Poisson's ratio during pruning. Contrary to the time consuming physics-based pruning approach from $\left[\mathrm{RPW}^{*} 18\right]$ we do not use the simulation at each iteration to monitor $v$ and to select the optimal edge to be removed. In order to monitor the evolution of $v$ during pruning we computed the series of intermediate networks from initial network $N_{0}$ to the final pruned network $N_{p}$, one network for each removed edge. The result for 5 representative networks with $Z=4.0$ is shown in Fig. 16 . The almost monotonically decreasing curves (from right to left) for decreasing $Z$ until 4.0, validates our geometric pruning approach. It is quite remarkable that a purely geometric criteria succeed so efficiently in finding edges with specific mechanical properties.

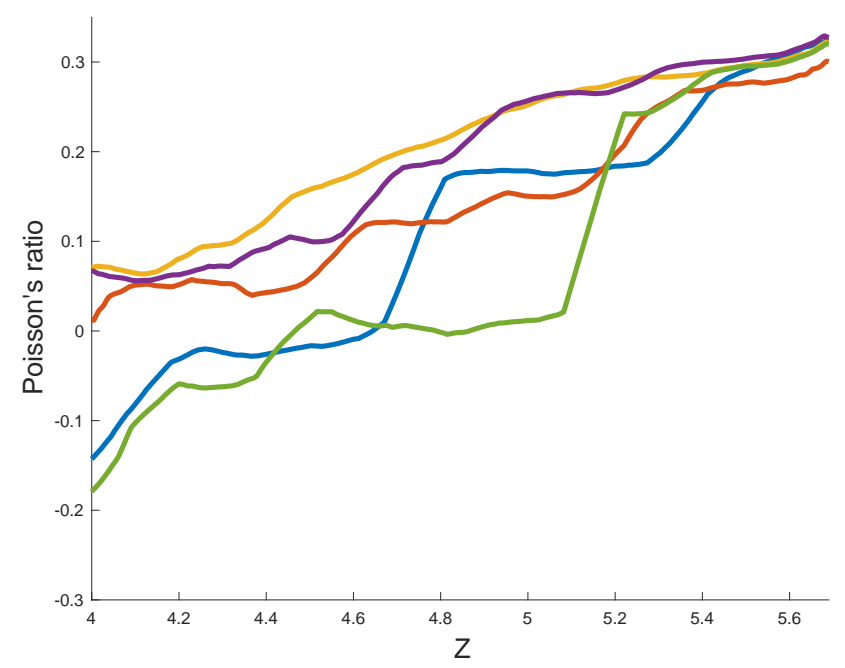

Figure 16: Evolution of Poisson's ratio: for 5 representative networks we computed 280 networks by iteratively pruning 280 edges and plot the curve of 280 Poisson ratios $v_{1}, \ldots, v_{280}$ for $\varepsilon_{y}=0.03$.

Index of pointedness. The index of pointedness is central to our method. We introduced this concept in order to develop an algorithm which monotonically increases the number of re-entrant vertices. The example shown in Fig. 12 (bottom) is representative for all networks and all $Z$ values. Indeed, we always observed the same pointedness ratios. The pruning algorithm was designed to reduce the index of pointedness of the network vertices, implying that not only the vertices' index decreases but consequently the number of re-entrant (0-pointed) vertices increases. The measured pointedness ratios together with improved Poisson's ratios confirm at least our initial observation about this geometric feature and the proper behavior of our pruning approach.

Random pruning without any strategy. As we have seen above, the vertex displacement method (spiky networks) for improving the pruned networks works well and is quite efficient. With the following experiment, we investigate the question : is the vertex displacement method efficient for any kind of networks, even for the dense initial ones? This would make our pruning method obsolete. This is indeed not the case. In Fig. 17 the vertex displacement method was applied to the initial network without any pruning as a representative example. As expected, the index of pointedness improves over the network vertices. Many vertices with index 2 become index 1 . However, the number of pointed vertices (green) does not increase. Again we have run the test on 100 networks. Evidence is given by the ratio of pointed vertices, which remains nearly the same: $8 \%$ for all the initial networks, $9 \%$ for the spiky versions. Also the Poisson's ratio stays quite stable. It slightly improves in average from $v=0.31$ to $v=0.26$, with low standard deviation, as shown in Fig. 17(e),(f) for all 100 networks.
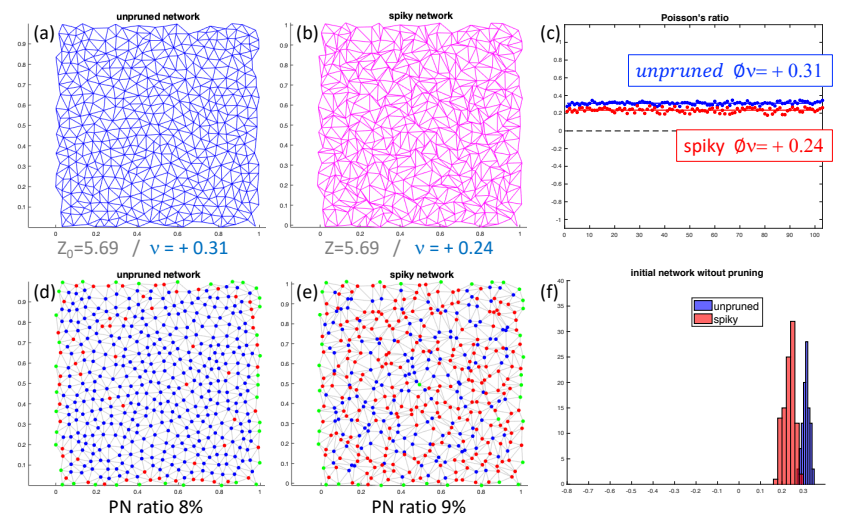

Figure 17: Spiky network generated from initial networks without pruning.
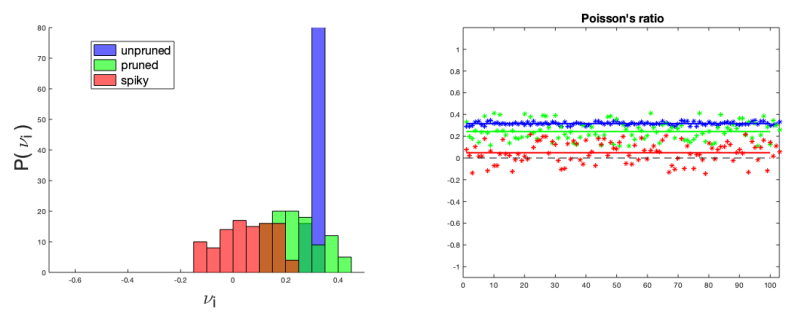

Figure 18: Random pruning: (left) Probability distribution of $\mathrm{v}$. (right) mean values of $\mathrm{v}_{\text {unpruned }}=0.31, v_{\text {pruned }}=0.24$ and $v_{\text {spiky }}=0.04$ for $\varepsilon_{y}=0.03$. Networks are mostly not auxetic.

Comparison to random pruning. It has been shown that pruning is an effective way to lower the Poisson's ratio to negative values. Since removing an edge reduces the degree of its two vertices by $1, Z$ is monotonically decreasing during pruning. A perhaps naive question is the following:

Is the criterion $Z<Z_{0}$ for a network sufficient to have a low Poisson's ratio?

In order to investigate this question experimentally, we implemented the naive pruning strategy of randomly removing edges. We computed 100 networks with $Z=3.9$ and made the following observations, see Fig. 18.

- The mean Poisson's ratio reduces merely from 0.3 to 0.24 thus indicating that random pruning is clearly not a sufficient strategy.

- We are however far from the $v$ values we can reach with the same coordination number with algorithm 1. 


\begin{tabular}{|c|c|c|c|c|}
\hline & & $Z=4.0$ & $Z=3.6$ & $Z=3.2$ \\
\hline$|V|$ & 458 & & & \\
\hline$|E|$ unpruned & 1356 & & & \\
\hline \#Edges removed & & 380 & 479 & 566 \\
\hline \multicolumn{5}{|c|}{ Timings in sec } \\
\hline preproc. PDS & & \multicolumn{3}{|c|}{0.48} \\
\hline preproc. DT & & \multicolumn{3}{|c|}{0.002} \\
\hline preproc. BE & & \multicolumn{3}{|c|}{0.023} \\
\hline Pruning & & 0.19 & 0.22 & 0.32 \\
\hline Spiky & & \multicolumn{3}{|c|}{0.24} \\
\hline Total & & 0.935 & 0.967 & 0.972 \\
\hline
\end{tabular}

Table 1: Statistics and execution times. $|V|$ number of vertices, $|E|$ number of edges, PDS Poisson Disk Sampling, DT Delaunay triangulation, BE removal of long boundary edges.

- Random pruning sometimes has the inverse effect: it increases $v$ above 0.3 with respect to the unpruned network.

- Optimizing a network with $Z<Z_{0}$ by making the vertices more spiky remains an efficient method to reduce $v$ whatever its value is.

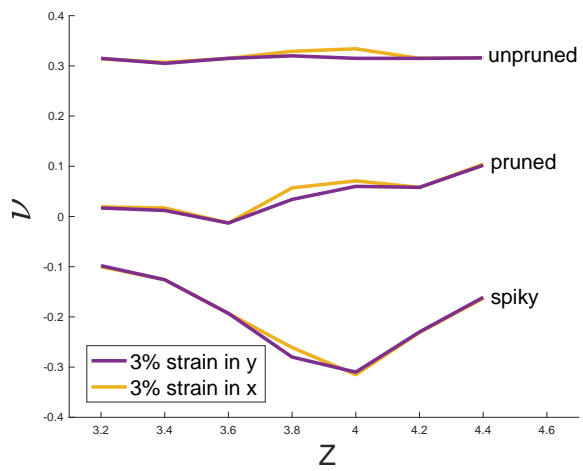

Figure 19: Test of isotropy: average Poisson's ratios computed with applied uni-axial strain of $3 \%$ in $x$ - and $y$-direction.

Isotropy. Isotropy is an important material feature. We compare the Poisson's ratios $v_{x}, v_{y}$ computed for a set of 100 networks (with 200 vertices) subject to uni-axial strain of 3\% in either $x$ or $y$-direction in Fig. 19. The curves for the 3 types of networks (unpruned. pruned, spiky) indicate that, in average, the networks are isotropic with respect to these two directions. This is coherent with the fact, that our geometric pipeline does not favor any specific orientation when selecting an edge for removal. However, individually, this is not always true for the pruned networks. Further investigation is necessary to examine isotropy with uni-axial experiments in arbitrary directions.

Execution times. Finally, we present some network statistics and CPU timings of the methods composing our approach. In total, our approach, including initial network computation, pruning with Algorithm 1 and improvement takes less than a second. Note that generation of input networks takes half of the time. The mass-spring simulation converges in less than $10 \mathrm{sec}-$ onds for our network sizes. We therefore compare favourably to the

physics-based pruning and simulation approaches which initially inspired our work.

When users seek a pruned network with a Poisson ratio close to $v=-0.35$ for example, the probability distribution in Fig. 14 indicates that they can choose among 10-20 networks within the corresponding bin [-0.33, -0.4]. In total, this procedure requires maximal 100 simulations.

Experimental realization. We follow the method from [RPW* 18$]$ for fabricating networks by laser-cutting rubber sheets. The material we choose is NBR Nitril because of its excellent resistance to break. It has a density of $1.45 \mathrm{~g} / \mathrm{m}^{3}$, a thickness of $2 \mathrm{~mm}$, and hardness of 65 Shores A. It is almost incompressible and has a Poisson's ratio $v=0.5$. These properties make this material ideal for our testing. Contrary to $3 \mathrm{D}$ printing of deformable microstructures, which requires to strengthen the joints by making them thicker [ $\left.\mathrm{PZM}^{*} 15\right]$, the opposite is required in our setting: we need to stiffen the edges away from the nodes in order to facilitate rotations at the nodes. This explains the polygonal geometry of our realized networks. We cut the rubber sheets with a TroTec 300 laser cutter. To produce the cutting paths, we compute offsets of the polygons in the network and reduce the width of the edges near nodes, as illustrated in Fig. 21.

This way we have fabricated 5 networks, produced by our algorithm, and laser-cut in a rubber sheet. Their photos are shown as insets in Fig. 20. Networks (a,b,d,e) are 40 cm large and have 203 vertices, network (c) is $20 \mathrm{~cm}$ large and has 110 vertices. All exhibit auxetic behavior in simulation, e.g. for extension of 3\% applied in either $x$ - or $y$-direction we get the following Poisson's ratios $v_{x}, v_{y}$ : (a)-0.36,-0.39, (b) $-0.47,-0.27$, (c) $-1.07,0.0$, (d) -0.87,-0.12, (e) $-0.92,0.13$. Fig. 1 shows three photos of network (a) taken from a compression and extension experiment with strain applied symmetrically in the $y$-direction. Fig. 22 (network (b)) shows another example where the loading applies only on the bottom boundary. The red network in the background corresponds to the rest state and the deformed networks are black.

In order to examine the matching between simulation and experiments, we conducted an experiment where we applied compression and tension strains of about $\pm 3 \%, \pm 6 \%$ and $\pm 10 \%$ to all 5 networks. We took a high-resolution photo and measured the real strains $\varepsilon_{x}$ and $\varepsilon_{y}$ on a pixel basis. The resulting Poisson's ratios for each network are plotted as orange dots in the graph in Fig. 20 and compared to the Poisson's ratios obtained through physical simulation by using the measured $y$-strains as boundary conditions. As a complement, Fig. 10 offers a visual matching for the teaser network. It can be observed that (1) all fabricated networks exhibit negative Poisson's rations and are thus auxetic. (2) The physical simulation method with the chosen tension and angular spring stiffness parameters provides estimates of the Poisson's ratio that are consistent with experiments. Note that for higher compression strains, our simulation is no longer able to accurately predict the node positions when they collide, thus resulting in lower values than in reality.

\section{Discussion \& Future work}

A limitation of our work is that it is restricted to planar structures. In future works, we would like to investigate an extension to $3 \mathrm{D}$ 


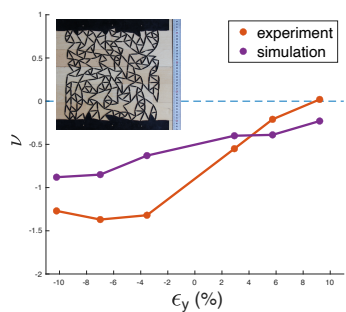

(a)

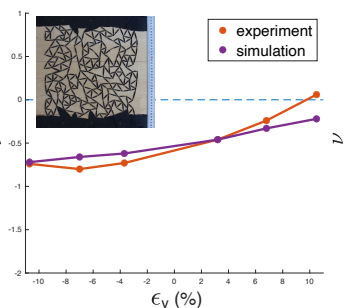

(b)

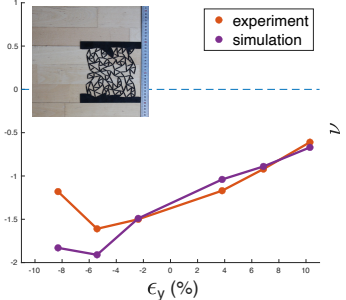

(c)

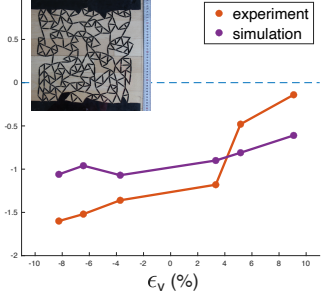

(d)

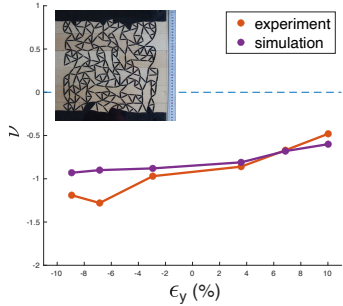

(e)

Figure 20: Comparison of Poisson's ratios estimated through physical simulation (purple dots) and measured (orange dots) for fabricated networks of a laser-cut rubber sheet (insets). Applied strains are close to $\pm 3 \%, \pm 6 \%$ and $\pm 10 \%$.
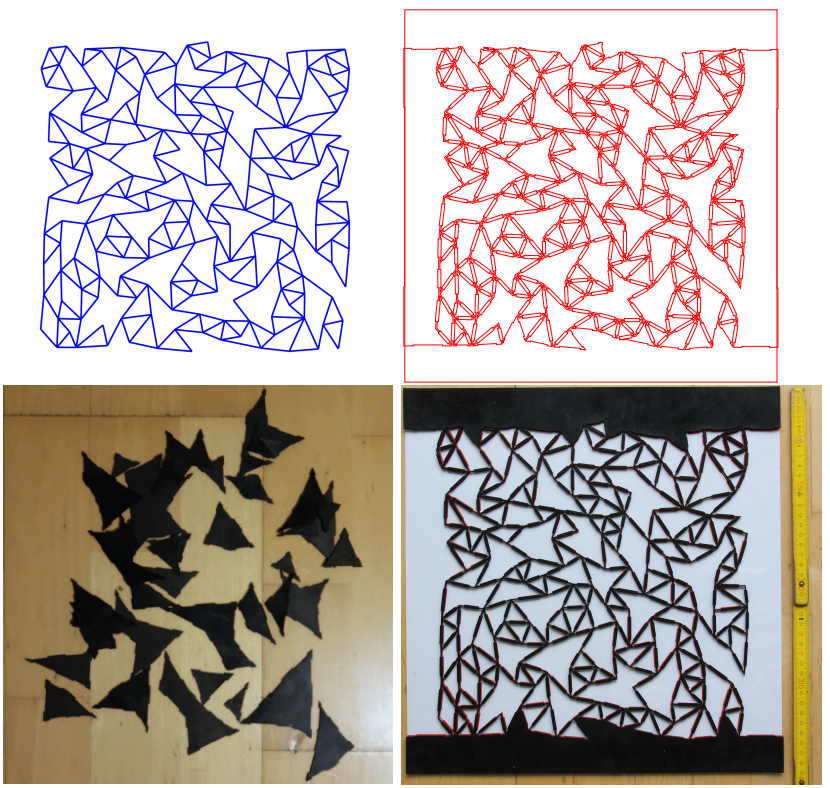

Figure 21: Producing laser-cut experimental realization of networks. Top-left: a network produced by our algorithm, Top-right: the actual cutting paths transmitted to the laser-cutter, Bottom-left: close-up on some of the polygons cut-out from the rubber sheet, Bottom-right: the resulting network.

irregular networks.

Gradient-based optimization techniques would probably strengthen the method. However, the discontinuous nature of an edge removal algorithm makes it difficult to formalize a differentiable cost function to be optimized by a gradient method. The vertex displacement could be solved this way and provide a globally more optimal solution than the implemented heuristic. However, optimizing a nonlinear function would increase computation time.

Our current implementation allows to apply uni-axial boundary conditions (BC) as well as bulk and shear BC. Implementation of periodic $\mathrm{BC}$ might enable us to scale-up and to conduct uni-axial stretching simulations in all planar directions and thus evaluate precisely the (an)isotropy of our designs.

As stated by Schumacher et al. [SMGT18], mechanical charac- terization of irregular tilings is not trivial. With our method we focused on material with one mechanical characteristic, the negative Poisson's ratio. Investigating a more deterministic method might offer insights on how to control additional mechanical parameters.

\section{Conclusion}

In this paper we have investigated the geometric construction of auxetic metamaterials with irregular networks. We analyzed important geometric features commonly found in irregular as well as regular auxetic networks and developed a pipeline relying solely on geometric criteria in order to produce irregular networks which exhibit these features. Since our method is based only on local geometrical criteria, it is less computationally intensive than related previous works based on physical simulations. We made extensive experiments validating our pipeline. We computed the Poisson's ratio of multiple sets of 100 networks with different characteristics produced with our pipeline, and showed that our pipeline effectively produces auxetic networks within a large range of negative Poisson's ratios.

Irregular designs can be attractive for potential applications in architecture, material science, furniture design, or homemade 3D printed materials with custom stretch. Our method offers a computational tool for constructing such new metamaterials.

\section{Acknowledgments}

This work was supported by the European Project ADAM2, (FETOpen, Grant agreement ID: 862025). The authors are grateful to Stan Borkowsky and the Amiqual4Home platform for giving us access to their laser-cut machine. We thank Mélina Skouras and JeanClaude Léon for helpful discussions.

\section{References}

[ACN20] Agnelli F., Constantinescu A., Nika G.: Design and testing of 3d-printed micro-architectured polymer materials exhibiting a negative poisson's ratio. Cont. Mech. Thermodyn. 32 (2020), 433-449. 3

[ALS14] Andreassen E., Lazarov B. S., Sigmund O.: Design of manufacturable $3 \mathrm{~d}$ extremal elastic microstructure. Mechanics of Materials 69, 1 (2014), 1 - 10. 3

[Bri07] BRIDSON R.: Fast poisson disk sampling in arbitrary dimensions. In ACM SIGGRAPH 2007 Sketches (New York, NY, USA, 2007), SIGGRAPH'07, Association for Computing Machinery. 5 

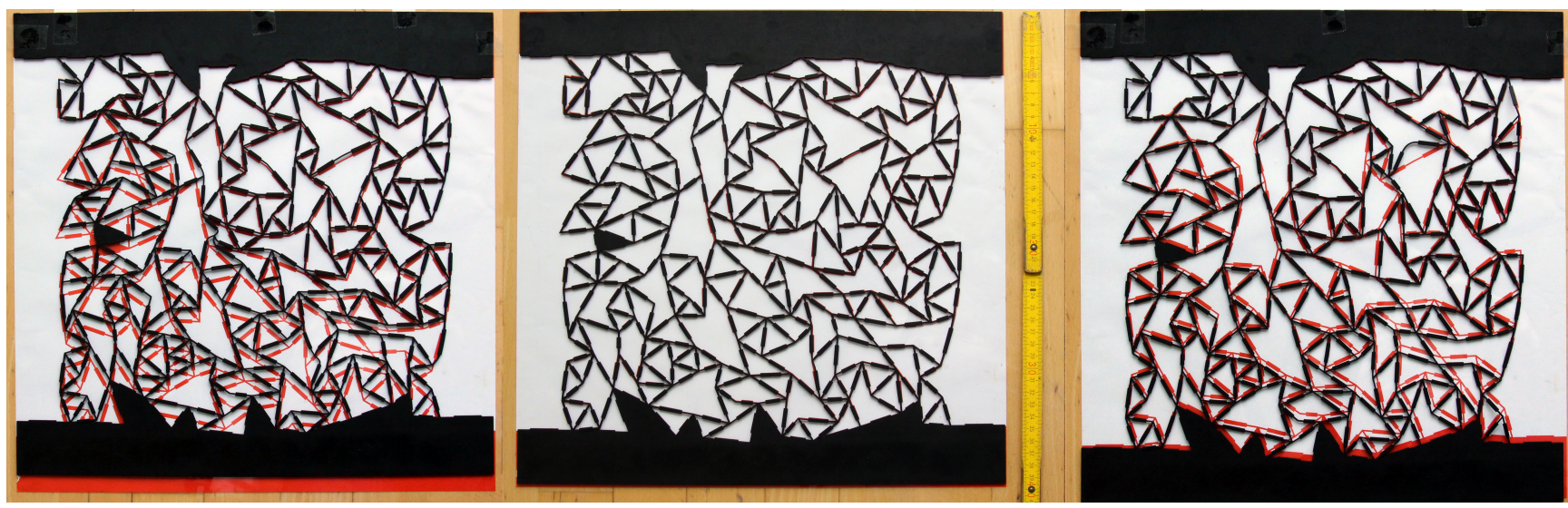

Figure 22: Vertical compression (left) and extension (right) of a laser-cut auxetic irregular network in rest state (middle). The compressed network exhibits a Poisson's ratio $\mathrm{v}=-0.32$ for applied strain $\varepsilon_{y}$ of $-3.4 \%$. The extended network exhibits a Poisson's ratio $\mathrm{v}=-0.36$ for applied strain $\varepsilon_{y}$ of $+2.1 \%$. Strains are applied to the bottom boundary.

[Cec21] CECIL T.: Auxetic table. https://tomcecil.co.uk, 2021. 2

[EL12] Elipe J. C. Á., LANTADA A. D.: Comparative study of auxetic geometries by means of computer-aided design and engineering. Smart Materials and Structures 21, 10 (2012), 105004. 2

[GGLR11] Greaves G. N., Greer A. L., LAKes R. S., RouXel T.: Poisson's ratio and modern materials. Nature Materials 10, 11 (2011), 823-837. 2

[GLN15] Goodrich C. P., LiU A. J., NAGEL S. R.: The principle of independent bond-level response: Tuning by pruning to exploit disorder for global behavior. Phys. Rev. Letters 114, 22 (2015). 2, 4

[HPaZ18] Hanifpour M., Petersen C., Alava M., Zapperi S.: Mechanics of disordered auxetic metamaterials. Eur. Phys. J. B 91, 10 (2018), 271. 3

[HSS*09] Horrigan E., Smith C., Scarpa F., Gaspar N., Javadi A., BERgER M., Evans K.: Simulated optimisation of disordered structures with negative poisson's ratios. Mechanics of Materials 41, $8(2009), 919-927.3,9$

[JTV*15] Jiang C., Tang C., Vaxman A., Wonka P., Pottmann H.: Polyhedral patterns. ACM Trans. Graph. 34, 6 (2015). 3

[KCD*16] Konaković M., Crane K., Deng B., Bouaziz S., Piker D., PAUlY M.: Beyond developable: Computational design and fabrication with auxetic materials. ACM Trans. Graph. 35, 4 (July 2016). 2,

[KLPCP18] Konaković-Luković M., Panetta J., Crane K., PAULY M.: Rapid deployment of curved surfaces via programmable auxetics. ACM Trans. Graph. 37, 4 (2018). 3

[KME*17] Kamrava S., Mousanezhada D., Ebrahimi H., GHOSH R., VAZIRI A.: Origami-based cellular metamaterial with auxetic, bistable, and self-locking properties. Scientific Reports 7 (2017), 46046. 2

[KZ17] Kolken H. A., ZadPOOR A.: Auxetic mechanical metamaterials. RSC Adv. 7 (2017), 5111-5129. 2

[Lak87] LAKES R.: Foam structures with a negative poisson's ratio. Science 235, 4792 (1987), 1038-1040. 2

[Lak91] LAKES R.: Deformation mechanisms in negative poisson's ratio materials: structural aspects. J. Material Science 26 (1991), 2287-2292. 8

[Lim17] Lim T.-C.: Analogies across auxetic models based on deformation mechanism. Phys Status Solidi RR 11, 6 (2017), 1-14. 2
[LN10] LiU A. J., NAgEL S. R.: The jamming transition and the marginally jammed solid. Annual Review of Condensed Matter Physics 1, 1 (2010), 347-369. 4, 5

[LNTX19] LIU J., NIE Y., TONG H., XU N.: Realizing negative poisson's ratio in spring networks with close-packed lattice geometries. Physical Review Materials 3 (05 2019). 4

[LWHZ14] LiU W., WANG N., HuAng J., ZHONG H.: The effect of irregularity, residual convex units and stresses on the effective mechanical properties of $2 \mathrm{~d}$ auxetic cellular structure. Materials Science and Engineering: A 609 (2014), $26-33.3$

[MCM*17] Mistry D., Connell S. D., Mickthwaite S. L., MorGan P. B., Clamp J. H., GleEson H. F.: Coincident molecular auxeticity and negative order parameter in a liquid crystal elastomer. Nat Commun 9, 5095 (2017). 2

[MDL16] Martínez J., Dumas J., Lefebvre S.: Procedural voronoi foams for additive manufacturing. ACM Trans. Graph. 35, 4 (July 2016).

[MPI*18] Malomo L., Pérez J., Iarussi E., Pietroni N., Miguel E., CignONi P., BiCKel B.: Flexmaps: Computational design of flat flexible shells for shaping 3d objects. ACM Trans. Graph. 37, 6 (2018).

[MSMB17] Mesa O., Stavric M., Mhatre S., Bechthold M.: Non-linear matters: Auxetic surfaces. In Proceedings of the 37th Annual Conference of the Association for Computer Aided Design in Architecture (Cambridge, USA, 2017), pp. 1-12. 2, 3

[MSS*19] Martínez J., Skouras M., Schumacher C., Hornus S., Lefebvre S., Thomaszewski B.: Star-shaped metrics for mechanical metamaterial design. ACM Trans. Graph. 38, 4 (July 2019).

[NM15] NABONI R., MiRante L.: Metamaterial computation and fabrication of auxetic patterns for architecture. Blucher Design Proceedings $\underline{2}, 3(2015), 129-136.2$

[OIBB*11] Omori T., Ishikawa T., Barthes-Biesel D., SalsaC A.-V., Walter J., Imai Y., Yamaguchi T.: Comparison between spring network models and continuum constitutive laws: Application to the large deformation of a capsule in shear flow. Physical review. E, Statistical, nonlinear, and soft matter physics 83 (04 2011), 041918. 8

[PMW*19] Peng R., Ma Y., Wu Q., Huang B., DaI Y.: Twodimensional materials with intrinsic auxeticity: progress and perspectives. Nanoscale 11 (2019), 11413-11428. 2 
[Poi33] PoIsson S.-D.: Traité de mécanique, tome 2. Bibliothèque nationale de France: https://gallica.bnf.fr/, 1833. 4

[PZM*15] Panetta J., Zhou Q., Malomo L., Pietroni N., CIGNONI P., ZoRIN D.: Elastic textures for additive fabrication. ACM Trans. Graph. 34, 4 (July 2015). 3, 11

[RDT*18] Ren X., Das R., Tran P., Ngo T. D., XIE Y. M.: Auxetic metamaterials and structures: a review. Smart Materials and Structures 27, 2 (jan 2018), 023001. 2, 4

[RPB*19] Reid D. R., PAshine N., Bowen A. S., NAgel S. R., DE PABLO J. J.: Ideal isotropic auxetic networks from random networks. Soft Matter 15 (2019), 8084-8091. 3, 4, 5

[RPW*18] Reid D. R., Pashine N., Wozniak J. M., Jaeger H. M., LiU A. J., NAgel S. R., DE Pablo J. J.: Auxetic metamaterials from disordered networks. Proceedings of the National Academy of Sciences 115, 7 (2018), 1384-1390. 2, 3, 4, 5, 10, 11

[SB07] Schlueter A., Bonwetsch T.: The m.any project - exploring a matrix model for a fully digital workflow in architectural design. In eCAADe 2007 (Frankfurt, 2007), p. 879-886. 3

[SB08] SCHLUETER A., Bonwetsch T.: Design rationalization of irregular cellular structures. International Journal of Architectural Computing 6 (2008), $197-211 . \overline{2}$

[SFS*19] Solly J., Früh N., SafFarian S., Aldinger L., MarGARITI G., KNIPPERS J.: Structural design of a lattice composite cantilever. Structures 18 (2019), 28 - 40. Advanced Manufacturing and Materials for Innovative Structural Design. 2

[SMGT18] Schumacher C., Marschner S., Gross M., THOMASZEWSKI B.: Mechanical characterization of structured sheet materials. ACM Trans. Graph. 37, 4 (2018). 2, 3, 12

[Sum19] Summivox: Poisson-disc sampling in matlab, 2019. https://summivox.wordpress.com/2015/09/19/ poisson-disk-sampling-in-matlab/. 8

[Tor13] TORONJO A.: Articles of apparel including auxetic materials. European Patent Office, EP2702884, August 2013. 2

[TTZ*20] Tricard T., TAVERnier V., ZAnni C., MartíneZ J., Hugron P.-A., Neyret F., LEFEBVRE S.: Freely orientable microstructures for designing deformable 3D prints. ACM Transactions on Graphics (Dec. 2020). 3

[VCW*17] Vogiatzis P., Chen S., Wang X., Li T., Wang L.: Topology optimization of multi-material negative poisson, Âôs ratio metamaterials using a reconciled level set method. Computer-Aided Design 83 (2017), 15 - 32. 3

[VV19] VINAY V. C., VARMA D. M.: Fabrication and testing of auxetic foams for rehabilitation applications. J Indian Inst Sci 99 (2019), 511-518. 2, 5

[WB06] Wächter A., Biegler L. T.: On the implementation of an interior-point filter line-search algorithm for large-scale nonlinear programming. Mathematical Programming 106, 1 (2006), 25-57. 7

[WSJ14] WANG F., Sigmund O., JENSEN J.: Design of materials with prescribed nonlinear properties. Journal of the Mechanics and Physics of Solids 69 (2014), $156-174.3$

[WVMr18] Wood D., Vailati C., Menges A., Rüggeberg M.: Hygroscopically actuated wood elements for weather responsive and self-forming building parts. facilitating upscaling and complex shape changes. Construction and Building Materials 165 (2018), 782 - 791. 2

[YY15] YASUDA H., YANG J.: Reentrant origami-based metamaterials with negative poisson's ratio and bistability. Physical review letters 114 (2015), 185502:1-5. 2

[ZEK*17] ZARRINMEhr S., ETtehad M., Kalantar N., Borhani A., Sueda S., AKLEMAN E.: Interlocked archimedean spirals for conversion of planar rigid panels into locally flexible panels with stiffness control. Computers \& Graphics 66 (2017), 93 - 102. Shape Modeling International 2017. 2
[ZJH16] Zhou L., JiAng L., HU H.: Auxetic composites made of 3d textile structure and polyurethane foam. physica status solidi (b) 253, 7 (2016), 1331-1341. 2

[ZK19] Zhang G., Khandelwal K.: Computational design of finite strain auxetic metamaterials via topology optimization and nonlinear homogenization. Computer Methods in Applied Mechanics and Engineering 356 (2019), $490-527.3$

[ZSCM17] Zhu B., Skouras M., Chen D., Matusik W.: Two-scale topology optimization with microstructures. ACM Trans. Graph. 36, 4 (July 2017). 3 